\title{
IMPORTANCIA RELATIVA DE LOS ACADÉMICOS Y DE LA ACADEMIA EN LA UNIVERSIDAD DE COSTA RICA (1988 - 1992)
}

\author{
Sonia Ma. Amador Berrocal
}

\section{Introducción}

Según el artículo 175 del Estatuto Orgánico de la Universidad de Costa Rica (UCR, 1990), los profesores tienen como principales funciones la enseñanza, la investigación y la participación activa en el desarrollo de los programas de acción social. Si se relacionan esas funciones del profesor, con la misión que la Universidad debe cumplir en la sociedad, es de esperar que los académicos ocupen un lugar preponderante, por ser ellos los responsables directos de ejecutar la misión universitaria.

Para determinar si existe o no coincidencia, entre la concepción teórica plasmada en el Estatuto Orgánico y la práctica institucional cotidiana, se tiene como propósito en el presente trabajo: establecer la importancia relativa que la Institución, en su accionar normal, ha concedido a los programas académicos y a los docentes, que no ocupan cargos docente-administrativos, encargados de la realización de las tres funciones básicas: docencia, investigación y acción social. El período considerado va de 1988 a 1992.

\section{Distribución del presupuesto de la Universidad de Costa Rica durante el quinquenio en estudio}

El FEES (Fondo Especial de la Educación Ferior! junto con las Transferencias Específi: $=$ CONARE. 1989) constituyen la Subven- General del Gobierno, a la que se suman antutarios e ingresos generados por cada institución, de acuerdo con lo estipulado en 1988 (La Gaceta No. 126, 1988), así como Asignaciones Especiales (Comisión de Enlace, s.f.), que financian a las cuatro instituciones de educación superior estatal. A pesar de esos otros ingresos mencionados, sigue siendo el FEES el principal aporte que financia a la educación superior pública (CONARE, Acta del $1 / 11 / 91$ ), sobre todo en la UCR, que durante la última década, ha visto disminuído el otorgamiento de Transferencias Específicas (CONARE, 1990).

Para un análisis como el que se desea hacer, existe la dificultad de que no se utiliza dentro de la Institución un único concepto de presupuesto. La Oficina de Contraloría Universitaria (OCU, 1992) define los siguientes tipos:

Presupuesto ordinario: corresponde al "proyecto de presupuesto" aprobado por el Consejo Universitario a fines del año anterior a su vigencia y el mismo incorpora la proyección de los ingresos que se espera obtener por concepto de la subvención por parte del estado, los recursos provenientes de leyes específicas, aportes de diversas instituciones tanto nacionales como del exterior y los llamados ingresos propios que son aquellos generados por la institución mediante la venta de bienes y servicios, lo cual incluye el cobro de la matrícula.

Presupuesto total: corresponde al "presupuesto ordinario" vigente para el respectivo período más la incorporación de todas las modificaciones intemas y externas del presupuesto tramitadas durante el mismo, las cuales se originan fundamentalmente por la presupuestación de nuevos recursos y el traslado de recursos de un programa a otro o bien dentro del mismo programa, en cuyo caso pueden producirse traslados de una unidad ejecutora a otra o de una partida presupuestaria a otra. El presupuesto total constituye el límite máximo autorizado de gastos $\mathrm{y}$, de conformidad con la normativa vigente establecida, no debe gastarse o comprometerse una suma que lo exceda. Presupuesto real: el presupuesto real corresponde al presupuesto realmente ejecutado (gastado o comprometido) 
durante el periodo. En buena teoría este no puede sobrepasar al "presupuesto total" que, como se indicó anteriormente, es el presupuesto máximo autorizado (OCU, 1992:1)

El problema mayor al analizar la situación presupuestaria de la UCR es que frecuentemente los documentos disponibles no indican cuál de las tres acepciones de presupuesto es la que están empleando y por ello muchas veces los datos no son concordantes.

Como punto de partida se inicia el análisis presupuestario de la UCR, comparando los ingresos anuales de la Institución, durante el quinquenio en estudio, por medio del Cuadro $\mathrm{N}^{\mathrm{N}} 1$.

Cuadro 1

Presupuesto anual de la UCR de 1988 a 1992, en colones y en dólares, considerando el tipo de cambio en cada año

\begin{tabular}{cccccc}
\hline Año & Colones & & Dólares & $\begin{array}{c}\text { Tipo de } \\
\text { cambio }\end{array}$ \\
\hline 1988 & 3 & 210000000 & 41 & 977246 & 76,47 \\
1989 & 3 & 754647600 & 45 & 833100 & 81,92 \\
1990 & 4 & 233000000 & 45 & 190563 & 93,67 \\
1991 & 5 & 739000000 & 48 & 029123 & 119,49 \\
1992 & 7 & 095000000 & 51 & 936169 & 136,61 \\
\hline
\end{tabular}

Fuentes: OPLAU, 1988 b; 1989 b; 1990; 1991; 1992.

Banco Central de Costa Rica.

Elaboración propia.
Ya que la variación en el tipo de cambio fue progresiva a lo largo del quinquenio y además existen diferencias entre la compra y la venta, el dato anual especificado para dicha tasa, se obtuvo haciendo un promedio entre los valores de compra y de venta, máximos y mínimos.

Las cantidades en colones experimentaron aumentos progresivos llegando a ser más del 100\% desde 1988 hasta 1992. Al considerar la devaluación, siempre creciente, sufrida por la moneda nacional ante el dólar estadounidense, los incrementos presupuestarios no son tan grandes, como puede observarse en el cuadro № 1 . En todo caso, ya sea en colones o en dólares, los presupuestos anuales siempre fueron en aumento a lo largo del quinquenio. Eso indica que en la UCR no ocurrió, entre 1988-92, el descenso de financiamiento que señalan diversos autores (Rojas, 1992; Rodríguez, 1992) para otras instituciones educativas nacionales y extranjeras, cuando el país es sometido a procesos de ajuste estructural.

La distribución del presupuesto universitario por Programas es reveladora en cuanto al grado de importancia que la misma Instinución concede a cada uno de ellos, de ahí que se prosiga con el correspondiente análisis.

Toda la cantidad de dinero que la UCR recibió, en distribución porcentual por Programas, durante el quinquenio de 1988 a 1992 y la proyección para 1993, aparece resumida en el cuadro $\mathrm{N}^{\mathrm{2}} 2$.

Cuadro 2

Distribución porcentual anual de egresos de la Universidad de Costa Rica por Programas, para el período 1988-1993

\begin{tabular}{lrrrrrr}
\hline Programa & 1988 & 1989 & 1990 & 1991 & 1992 & 1993 \\
\hline & & & & & & \\
Docencia & 45,80 & 40,33 & 37,56 & 36,37 & 38,36 & 40,87 \\
Investigación & 13,24 & 13,94 & 13,48 & 13,26 & 12,27 & 12,61 \\
Acción Social & 2,32 & 2,68 & 2,62 & 2,78 & 2,30 & 2,46 \\
Vida Estudiant. & 7,53 & 7,35 & 6,37 & 5,56 & 5,48 & 5,92 \\
Administración & 16,55 & 20,95 & 16,77 & 19,31 & 17,27 & 12,96 \\
Direc. Superior & 12,70 & 13,05 & 12,23 & 12,99 & 13,07 & 13,38 \\
Desarr. Regional & - & 1,70 & 8,97 & 8,57 & 9,25 & 9,93 \\
Inversiones & 1,87 & & 2,00 & 1,15 & 1,99 & 1,87 \\
\hline
\end{tabular}

Fuentes: OCU, 1992; OPLAU, 1993. 
En relación con el presupuesto de 1992, el incremento general que experimentó la UCR en 1993, fue de un $25 \%$. Esto en números absolutos significó una diferencia de $\$ 1.779$ millones, pues se aumentó de $\$ 7.095$ millones en 1992, a $\$ 8.874$ millones en 1993 (OPLAU, 1993).

Para efectos del presente trabajo, con base en la información suministrada en el cuadro № 2, interesa destacar que el Programa de Docencia consumió el porcentaje mayor del presupuesto universitario, aunque éste disminuyó su participación a lo largo del quinquenio. El Programa de Investigación ocupó un tercer lugar de 1988 a 1991, pero pasó al cuarto lugar en 1992 y 1993. La Acción Social tuvo una importancia relativa muy baja dentro del gasto, puesto que se encuentra en el penúltimo lugar. Según indicó el Jefe de Presupuesto de la Oficina de Planificación Universitaria (Sánchez, 1993), los cambios operados en la distribución interna del presupuesto universitario, son el resultado de las recomendaciones emanadas de las unidades académicas en conjunto con las Vicerrectorías, quienes en primera instancia elaboran propuestas, que son finalmente aprobadas por el Consejo Universitario.

Analizando la situación desde otro ángulo. en este caso según el personal que labora para cada uno de los Programas, puede deducirse que a pesar de consumir Docencia el mavor porcentaje del presupuesto, y ser allí $\vdots$ - de se agrupa el grueso de los docentes, no :-z precisamente el personal académico el  $\therefore-$ de seguido.

E- ia LCR el personal se clasifica en tres $=0$ grupos, según la función que desemĐé_e_efiniéndose así: (OPLAU, 1989 a: 5)

$\rightarrow$ Cic Jente (D): son los funcionarios universitarios Te ra paipal quehacer, tienen a su cargo la ense-

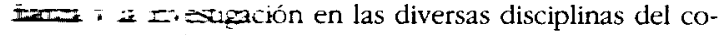
IIIOHe: : i zarkipación activa en los programas de torir sin

Iex- de esta categoría se incluyen, paI Iezis fresupuesto, los tiempos com-

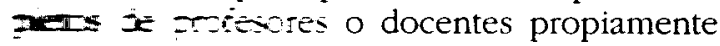
zacs inter las horas estudiante $\pi=-100=0$
Personal de apoyo académico (AA): se refiere al personal que ocupa una serie de plazas cuyo objetivo y funciones evidencian su carácter de apoyo a las actividades de Docencia, Investigación, Acción Social y Orientación Estudiantil.

Personal administrativo (A): son los que tienen a su cargo funciones complementarias a las actividades de Docencia, Investigación y Acción Social.

Ya que en la práctica tienden a confundirse los puestos de apoyo académico con los administrativos, se consultó el Presupuesto por Programas y Actividades 1988 (OPLAU, 1988 b), encontrándose que tampoco hay diferencias importantes de salarios entre ellos. Dado que todo ese personal no ejecuta directamente la docencia, la investigación, o la acción social, para efectos del presente trabajo se considerarán ambos tipos en un solo grupo: el de los administrativos. El personal docente está formado sólo por aquellos que ejecutan al menos una de las tres funciones básicas en la Institución. En forma distinta, la Universidad considera dentro de la categoría denominada "plazas académicas", al conjunto formado por las plazas docentes más las plazas de apoyo académico (OPLAU, 1990).

Datos de CONARE (1992) revelaron que el $82 \%$ del personal académico se encontraba ubicado en el Programa de Docencia, situación que responde a lo establecido en el inciso e del Reglamento de Régimen Académico y Servicio Docente (UCR, 1993).

Algunos académicos dedican parte de su carga a otras actividades como la investigación o la labor docente administrativa, cuyo porcentaje no puede inferirse de la información hallada. Además, debe recordarse que los puestos de apoyo académico también se encuentran incluídos en esta categoría, según la aclaración que se hiciera en un párrafo anterior.

Los administrativos se ubicaron principalmente en los Programas de Docencia, de Administración y de Investigación, en ese orden; el Programa de Acción Social es el que reúne los menores porcentajes de personal tanto administrativo como docente (CONARE, 1992). En el caso de Investigación, la cantidad de docentes es comparativamente muy reducida $(6 \%)$, puesto que, como se indicó antes, la mayoría de los investigadores están incluidos en el Programa de Docencia. 
A continuación se agrupa la distribución de las plazas de tiempo completo ocupadas en la Sede Rodrigo Facio por el personal docente, de apoyo académico y administrativo, para los años de 1989 a 1993; además, se especifica el número de tiempos completos (T.C.) ocupados por los profesores o docentes. Los datos se recopilan en el cuadro № 3 .

A pesar de que entre el grupo de plazas docentes se encuentran las cifras más elevadas, es importante recordar que este rubro incluye las plazas de horas profesor, horas asistente, horas estudiante y docentes o plazas de profesor (T.C. de Prof.). Estos últimos aparecen en el renglón final del cuadro № 3 en el que puede notarse un descenso sensible de las cantidades, resultando el número de plazas docentes de tiempo completo, menor al de plazas administrativas.

Del análisis más detallado de la distribución de cargas académicas, en horas por semana, que se encuentra agrupada en el cuadro № 4, es posible determinar que de 1988 a 1992 el porcentaje de carga en Docencia disminuyó en 8,40 puntos y en Acción Social en 0,02. Para los Programas de Investigación, Docente-administrativa y Comisiones Institucionales y otros, por el contrario, aumentó el número de puntos en $2,09,3,70$ y 2,65 respectivamente. Las cifras indican que conforme se da el crecimiento en estos tres últimos Programas, disminuye Docencia.

Con los datos del cuadro № 4 se evidencia que los Programas Docente-Administrativo

Cuadro 3

Distribución anual de plazas

equivalentes a tiempo completo, de 1989 a 1993

\begin{tabular}{lrrrrr}
\hline Plazas de T.C. & 1989 & 1990 & 1991 & 1992 & 1993 \\
\hline & & & & 2112,194 & 2112,194 \\
Docentes & 2139,587 & 2112,174 & 2112,174 & 1934,238 & 634,238 \\
Administrat. & 1914,738 & 1934,238 & 628,181 & 624,125 & 1824,852 \\
Apoyo Acad. & 625,500 & 628,813 & 1819,565 & 1824,852 \\
T.C. de Prof. & 1815,127 & 1819,565 & & 1825 \\
\hline
\end{tabular}

Fuentes: OPLAU, 1988 b; 1989 b; 1990; 1991; 1992; 1993.

Elaboración propia.

Cuadro 4

Distribución de la carga académica de la UCR por Programas durante el quinquenio 88-92, en términos relativos de horas por semana

\begin{tabular}{|c|c|c|c|c|c|}
\hline \multicolumn{6}{|c|}{ Programas } \\
\hline Año & Docen. & Invest. & Acc. Soc. & Doc. Adm. & $\begin{array}{c}\text { Comis.Inst. } \\
\text { y otros }\end{array}$ \\
\hline 1988 & 72,86 & 9,78 & 3,01 & 11,80 & 2,53 \\
\hline 1989 & 69,65 & 10,61 & 2,85 & 11,98 & 4,89 \\
\hline 1990 & 66,52 & 11,64 & 3,18 & 12,73 & 5,92 \\
\hline 1991 & 65,08 & 11,25 & 3,02 & 15,47 & 5,15 \\
\hline 1992 & 64,46 & 11,87 & 2,99 & 15,50 & 5,18 \\
\hline Dif. & $-8,40$ & $+2,09$ & $-0,02$ & $+3,70$ & $+2,65$ \\
\hline $88-92$ & & & & & \\
\hline
\end{tabular}

Fuentes: CEA, 1993.

Elaboración propia. 
y Comisiones Institucionales y otros, fueron los que crecieron mayormente, sumando en conjunto 6,35 puntos desde el inicio hasta el fin del quinquenio comprendido, en contraposición a los 2,09 puntos aumentados por la Investigación, en ese período. Por lo tanto, la disminución en la carga docente debida probablemente al menor número de estudiantes en la Institución, se presume que fue en favor de la burocracia universitaria.

Si el presupuesto total incluye modificaciones al presupuesto ordinario, debidas a la incorporación de nuevos recursos y al traslado de partidas de un programa a otro o dentro del mismo programa, es importante analizar el comportamiento de esos cambios presupuestarios. Para ello se elabora el cuadro № 5 .

Cuadro 5

Diferencias de participación porcentual anual entre los presupuestos ordinario y total, por Programas, de 1989 a 1992

\begin{tabular}{lrrrr}
\hline & Programa & 19891990 & 1991 & 1992 \\
\hline & & & & \\
Docencia & $-0,86$ & $+1,72$ & $-3,80$ & $-2,10$ \\
Investigación & $-0,03$ & $+1,12$ & $+0,43$ & $-0,10$ \\
Ac. Social & $+0,12$ & $+0,45$ & $+0,41$ & $-0,18$ \\
Vida Estud. & $-0,74$ & $-0,54$ & $-1,07$ & $-0,71$ \\
Administr. & $+1,18$ & $-2,86$ & $+5,83$ & $+3,96$ \\
Dir. Superior & $+0,57$ & 0 & $-0,39$ & $-0,53$ \\
Des. Regional & 0 & $+0,17$ & $-1,15$ & $-0,58$ \\
Inversiones & $-0,24$ & $-0,06$ & $-0,29$ & $+0,59$ \\
& & & & \\
\hline
\end{tabular}

Fuentes: OCU, 1990; 1992.

Elaboración propia.

Puede observarse que los programas de Docencia, Vida Estudiantil e Inversiones, se modificaron negativamente excepto en 1990 el primero y en 1992 el segundo. Esto indica una merma conforme se pasa del presupuesto ordinario al total o expresado en otra forma, segun se introducen modificaciones en el presupuesto aprobado originalmente por el Consejo Lriversitario. Sobre todo el primero de ellos, ¿ Docencia, sufrió las mayores disminuciones ¿E : odo el cuadro, durante los años 91 y 92 . A : : Programas de Investigación y de Acción S.:z1. Las modificaciones introducidas unas Zies los favorecen y otras no. En Administra- ción, principalmente en los últimos dos años, las diferencias positivas fueron tan altas al modificarse el presupuesto, que no tienen punto de comparación con ningún otro Programa.

Curiosamente los cambios operados en los presupuestos de los Programas de Docencia y Administración son opuestos, pues cuando el primero baja el segundo sube y viceversa. En los periodos 89 y 90 se dio un equilibrio en las modificaciones introducidas, positivas y negativas, para los diferentes Programas. En los períodos 91 y 92 fueron mermados los recursos para la mayoría de los Programas, viéndose favorecidos solamente dos o tres, entre los que destacan notoriamente las diferencias positivas de 5,83 y 3,96 para Administración.

Este análisis sugiere que las acciones presupuestarias ejecutadas en la UCR favorecieron a las actividades burocrático-administrativas, en detrimento de los Programas de Docencia. Tampoco esta política presupuestaria parece haber estimulado a la Investigación o la Acción Social en forma proporcional y conveniente, como lo dicta su Estatuto Orgánico.

Otro de los factores importantes de revisar es la proporción del presupuesto destinado al pago de salarios propiamente, y la que se invirtió en gastos de otro tipo, pues como indica Reimers (1989), la tendencia al crecimiento de la primera proporción es un indicador que se pone de manifiesto en la educación pública superior de países latinoamericanos, cuando éstos tienen que hacerle frente al gran endeudamiento externo que obliga a la implementación de Programas de Ajuste Estructural.

En la UCR, durante 1990, el Consejo Universitario se pronunció en procura de que el porcentaje del presupuesto correspondiente al pago de salarios (denominado "masa salarial"), se mantuviera dentro de ciertos límites. En su sesión № 3761, en agosto de 1991, dicho Consejo acordó mantener la masa salarial en un $78 \%$ del presupuesto universitario y que el resto de las partidas o "partidas generales", alcanzara al menos un 22\% (OCU, 1992).

Los porcentajes del presupuesto universitario correspondientes a masa salarial y partidas generales, incluidos en los tres tipos de presupuesto (ordinario, total y real), desde 1989 hasta 1992, aparecen reunidos en el cuadro № 6 . 
Cuadro 6

Porcentajes anuales de masa salarial y partidas generales en los presupuestos ordinario, total y real,de 1989 a 1992

\begin{tabular}{lcccccccc}
\hline & \multicolumn{2}{c}{1989} & \multicolumn{2}{c}{1990} & \multicolumn{2}{c}{1991} & \multicolumn{2}{c}{1992} \\
Presup. & M.Sal. & P.Gen. & M.Sal. & P.Gen. & M.Sal. & P.Gen. & M.Sal. & P.Gen. \\
\hline & & & & & & & & \\
Ord. & 75,12 & 24,88 & 74,14 & 25,86 & 75,40 & 24,60 & 80,03 & 19,97 \\
Total & 74,18 & 25,82 & 77,69 & 22,31 & 75,35 & 24,65 & 78,20 & 21,80 \\
Real & 75,78 & 24,22 & 79,12 & 20,88 & 78,25 & 21,75 & - & - \\
\hline
\end{tabular}

Fuente: OCU, 1992

Se observa que el porcentaje de participación de la masa salarial (M.Sal.) aumentó, mientras el de partidas generales (P.Gen.) disminuyó, especialmente en el año 1990. Para 1991, probablemente debido a la influencia del acuerdo tomado por el Consejo Universitario que se mencionó anteriormente, los porcentajes se acercaron bastante a los acordados, pero aún así siempre se desbalanceó a favor de la masa salarial.

Aunque no está completo el año 1992, ya el presupuesto total indica que se sobrepasó la cifra acordada y es de esperar que también esa relación siguiera los mismos pasos del año anterior, puesto que los datos incluidos en el cuadro cuadro № 6 muestran un aumento en el porcentaje de participación de la masa salarial, conforme se pasa del presupuesto ordinario al total, y de éste al real, excepto en 1989. Por lo demás, siendo el presupuesto real el que se ejecuta, puede afirmarse que presupuestariamente la Institución introdujo modificaciones no programadas, tal vez debido a aumentos de salarios al personal, no previstos y que además invirtió una parte cada vez mayor del presupuesto, en el renglón de masa salarial.

Tal situación calza con el pensamiento ya mencionado de Reimers (1989), quien asegura que el fenómeno se presenta en la educación superior pública, cuando el gasto en educación disminuye, conforme aumenta el endeudamiento del país.

\section{Análisis de las Vicerrectorias}

A continuación se procede a analizar la distribución presupuestaria de cada una de las Vicerrectorías de Docencia, de Investigación y de Acción Social, como entes encargados de ejecutar las tres funciones básicas de la Universidad.

\section{II. a. Vicerrectoria de Docencia}

La Vicerrectoría de Docencia es un órgano ejecutivo, asesor y coadyuvante de la Rectoría. La actividad docente en la Universidad está regida por esta Vicerrectoría, dependiendo de ella administrativamente todas las unidades académicas, el Centro de Evaluación Académica y los programas de: Cargas Académicas, Evaluación de Planes de Estudio, Evaluación del Personal Docente y Evaluación Institucional (CEA, 1981).

Tal como se indicara anteriormente, el programa ejecutado por esta Vicerrectoría es el que recibe el mayor porcentaje del presupuesto universitario y a ella pertenecen todos los docentes que a la vez desempeñan actividades de investigación, acción social y administración, lo cual le concede una gran importancia y torna difícil el análisis individual de sus actividades. 


\section{Cuadro 7}

Distribución porcentual anual del presupuesto de Docencia por Subprogramas para el período de 1989 a 1993

\begin{tabular}{|c|c|c|c|c|c|}
\hline Subprograma & 1989 & 1990 & 1991 & 1992 & 1993 \\
\hline Apoyo a Docencia & 7,48 & 6,65 & 16,02 & 9,49 & 9,18 \\
\hline Anes y Letras & 10,38 & 10,61 & 9,52 & 10,47 & 10,47 \\
\hline Ciencias Básicas & 14,93 & 14,86 & 13,48 & 14,48 & 14,24 \\
\hline Cienc. Sociales & 25,01 & 25,41 & 13,09 & 24,60 & 24,89 \\
\hline Salud & 21,93 & 22,00 & 19,45 & 21,05 & 20,81 \\
\hline Ing. y Arquitec. & 14,78 & 14,96 & 13,56 & 14,68 & 15,01 \\
\hline Estud. Generales & 4,83 & 4,83 & 4,35 & 4,71 & 4,89 \\
\hline Emp. Aux de Doc. & 0,65 & 0,69 & 0,54 & 0,53 & 0,51 \\
\hline
\end{tabular}

Fuente: OPLAU, 1993.

Tratando de determinar cuáles fueron las áreas que consumieron la mayor parte de recursos de este Programa, se analiza en el cuadro $\mathrm{N}^{\mathrm{T}} 7$ la distribución interna del presupuesto de Docencia.

Los subprogramas correspondientes a áreas académicas, según la importancia que se les concedió en el presupuesto, se ordenan así: Ciencias Sociales, Salud, Ingeniería y Arquitectura junto con Ciencias Básicas, Artes y Letras y finalmente Estudios Generales.

Si se ignora el caso especial de 1991, se experimentó a lo largo del quinquenio un descenso en la participación de los subprogramas de Ciencias Básicas, Ciencias Sociales, Salud y Empresas Auxiliares de Docencia. Permanecieron más o menos estables Artes y Letras y Esrudios Generales. El subprograma de Ingenieria y Arquitectura incrementó tímidamente su porcentaje de participación, lo mismo que el de Apoyo a la Docencia, pero éste en forma nis agresiva.

En términos generales, a lo largo del zunquenio no se evidencia cambio en el orJen de importancia que se concede a las difeE-z:e áreas académicas, lo que sugiere que trrante el periodo en estudio, hubo muy po$2=$ modiricaciones en este sentido, para adapI- Li Liversidad a las nuevas corrientes ideoaxas neoliberales, según señalan algunos zteres "Camacho. 1990).

Ë Supprograma de Apoyo a Docencia de ise icerrectoria, como su nombre lo inare in crresponde a ninguna área académi- ca sino que ofrece un respaldo a éstas. De éste conviene resaltar, a partir del cuadro $N^{\circ} 7$, el fenómeno ocurrido en 1991: su porcentaje de participación aumentó en más del doble, en comparación con el año anterior, mientras que el resto de subprogramas disminuyó. Para Ciencias Sociales fue tan drástico el descenso en ese mismo período, que casi se redujo a la mitad. Aunque en 1992 se trató de retornar a la situación anterior, siempre el Apoyo a Docencia experimentó una alza considerable en los años sucesivos, que no tiene par en ningún otro subprograma.

El subprograma Apoyo a Docencia incluye las siguientes actividades: Licencia Sabática (Lic. Sabát.), Centro de Evaluación Académica (C.E.A.), Tercer Ciclo (3er. Ciclo), Programas Autofinanciados (Prog. Autof.), Servicios de Apoyo de Docencia (Serv. Apoyo Doc.) y Recursos Humanos Especializados (Rec. Hum. Esp.) (OPLAU, 1991). Para analizar el fenómeno que se presentó en este subprograma durante 1991, señalado anteriormente, se desglosa en el cuadro № 8 , la subdivisión que se hizo de su presupuesto, a lo largo del quinquenio.

En 1991 experimentó una brusca caída la importancia presupuestaria del CEA. En menor medida, pero en relación también importante, disminuyeron los presupuestos destinados a Licencia Sabática, Programas Autofinanciados y Tercer Ciclo, aunque este último ya había sido sensiblemente mermado desde el año anterior. Apareció por primera vez la actividad Recursos Humanos Especializados 
Cuadro 8

Distribución porcentual anual del presupuesto del Subprograma Apoyo a Docencia, en sus seis actividades, de 1988 a 1992

\begin{tabular}{|c|c|c|c|c|c|}
\hline Actividad & 1988 & 1989 & 1990 & 1991 & 1992 \\
\hline Lic. Sabát. & 3,71 & 3,75 & 5,77 & 2,19 & 3,99 \\
\hline C.E.A. & 20,06 & 20,33 & 23,04 & 8,48 & 14,84 \\
\hline 3er. Ciclo & 19,46 & 19,63 & 3,08 & 1,16 & 2,11 \\
\hline Prog. Autof. & 7,46 & 7,37 & 8,02 & 2,46 & 3,49 \\
\hline Serv. Apoyo Doc. & 49,28 & 48,91 & 60,08 & 84,24 & 72,90 \\
\hline Rec. Hum. Esp. & - & - & & 1,46 & 2,66 \\
\hline
\end{tabular}

Fuentes: OPLAU, 1988 b; 1989 b; 1990; 1991; 1992

Elaboración propia.

Cuadro 9

Distribución porcentual anual del presupuesto de Docencia correspondiente al pago de salarios, según los tipos de empleados

\begin{tabular}{crrrrr}
\hline Personal & 1988 & 1989 & 1990 & 1991 & 1992 \\
\hline \multirow{2}{*}{ D } & 83,26 & 79,80 & 77,94 & 79,80 & 79,33 \\
A & 15,33 & 3,21 & 14,51 & 13,27 & 13,67 \\
A A & 1,41 & 6,98 & 7,54 & 6,91 & 6,99 \\
A + AA & 16,74 & 20,19 & 22,05 & 20,18 & 20,66 \\
\hline
\end{tabular}

Fuentes: OPLAU, 1988 b; 1989 b; 1990; 1991; 1992.

Elaboración propia.

con un porcentaje de participación bastante bajo. El Servicio de Apoyo a Docencia fue el único que se elevó en más de un $20 \%$, con respecto al año anterior y casi se duplicó en comparación con 1988 y 1989. Los datos indican para 1992 una recuperación de las otras actividades, a la vez que desciende el porcentaje de Servicios de Apoyo a Docencia. Puede afirmarse que el subprograma de Apoyo a Docencia elevó, en una forma sin precedentes, su importancia presupuestaria durante 1991 , debido al incremento experimentado en la actividad Servicios de Apoyo de Docencia. Al revisar qué incluye dicha actividad (OPLAU, 1990) se presenta de nuevo la circunstancia discutida anteriormente, de que no existe una clara diferenciación entre estos puestos y los administrativos, de manera que el alza en esta actividad y por ende del subprograma Apoyo a Docencia, se debió al incremento de la parte administrativa, en detrimento de otras actividades como Licencia Sabática y de otros subprogramas, que involucran directamente a la parte académica.

En cuanto a la porción del presupuesto de Docencia correspondiente al pago de salarios, éste se distribuyó entre el personal docente (D), el administrativo (A) y el de apoyo académico (AA), tal como aparece, en datos porcentuales, en el cuadro № 9 .

El grueso del presupuesto de Docencia correspondiente al pago de salarios, se dedicó al personal docente, lo cual es congruente con el elevado porcentaje de empleados docentes que allí se congregan, según se había establecido previamente.

De 1988 a 1989 se observa un descenso considerable en la participación salarial de los primeros dos grupos considerados, que no se 
recupera a lo largo del quinquenio, pero aumenta el porcentaje destinado al personal de apoyo académico. En 1990, mientras desciende aún más el porcentaje para docentes, aumentan los de administrativos y apoyo académico. La situación retorna en 1991 a un estado muy parecido al de dos años atrás y así se mantiene para 1992. El presupuesto para apoyo académico aumenta a costas del destinado a docentes, en primera instancia, y en segunda instancia del de administrativos.

En el último renglón del cuadro anterior se aplica el criterio establecido previamente, de considerar en un solo conjunto al personal administrativo y al de apoyo académico, pues ninguno de esos ejecuta funciones de docencia, investigación o acción social.

Al comparar ese grupo con el personal docente, a pesar de que éstos siempre predominan, disminuyen su importancia relativa a lo largo del quinquenio, mientras el conjunto del personal administrativo y el de apoyo académico, la aumentan.

Relacionando este análisis de distribución presupuestaria según el personal, con el anterior de distribución presupuestaria por subprogramas y actividades, se observa que hay coincidencia en el crecimiento relativo experimentado por el personal no académico y por el subprograma de Apoyo a Docencia. Este incremento se lleva a cabo sacrificando primordialmente el porcentaje correspondiente a los docentes y a las áreas académicas.

La interpretación de los datos conduce a pensar en disposiciones que favorecen el presupuesto destinado a lo administrativo, en detrimento de lo académico propiamente dicho. Ese cambio es el que se destaca a partir del análisis presupuestario de la Vicerrectoría de Docencia.

\section{I. b. Vicerrectoria de Investigación}

La Vicerrectoría de Investigación es un órgano ejecutivo, asesor y colaborador de la Rectoria (CEA, 1981). Para que esta Vicerrectoría pueda cumplir con la misión encomendada. debe impulsar y apoyar prioritariamente los provectos y programas inter y multidisciplinarios. para lo cual cuenta con la asistencia de una serie de unidades especializadas en ac- tividades investigativas, que se clasifican en: Institutos de Investigación, Centros de Investigación y Estaciones Experimentales (UCR, 1979).

Las funciones de divulgación de conocimientos que debe llevar a cabo la Vicerrectoría, son ejecutadas por medio de la Dirección Editorial y de Difusión de la Investigación (DIEDIN), que se encarga de difundir los resultados de las investigaciones. Además cuenta con la edición de 14 revistas especializadas, libros de texto y otros, mediante los cuales logra poner al alcance de los usuarios, las investigaciones realizadas.

El presupuesto asignado para el cumplimiento de las funciones mencionadas, disminuyó desde $13,24 \%$ del presupuesto universitario de 1988 (OCU, 1992), hasta $12,62 \%$ en 1993 (ver cuadro № 2).

Al presupuesto de Investigación se suman anualmente, otros recursos provenientes de organismos financieros nacionales e internacionales, que respaldan proyectos específicos.

La subdivisión interna del presupuesto del Programa de Investigación, de 1988 a 1992, por subprogramas, da una idea de la importancia relativa de cada uno de ellos. Los datos aparecen tabulados en el cuadro № 10 .

\section{Cuadro 10}

Distribución porcentual anual del presupuesto total de Investigación, por subprogramas, de 1988 a 1992

\begin{tabular}{lrrrrr}
\hline Subprograma & 1988 & 1989 & 1990 & 1991 & 1992 \\
\hline & & & & & \\
Apoyo a Invest. & 56,95 & 53,26 & 57,74 & 60,85 & 57,05 \\
Cen. e Inst. Invest. & 33,50 & 32,43 & 35,77 & 34,51 & 37,94 \\
SEP & 3,29 & 3,15 & 3,57 & 3,61 & 3,91 \\
Emp. Aux. Invest. & 6,24 & 6,30 & 2,89 & 1,02 & 1,09 \\
Sedes Regionales & - & 4,83 & - & - & - \\
\hline
\end{tabular}

Fuentes: OPLAU, 1988 b; 1989 b; 1990; 1991; 1992. Elaboración propia.

Se repite en este programa la misma situación del presupuesto de Docencia: el subprograma de Apoyo a la Investigación (Apoyo a Invest.) es el que tiene mayor importancia relativa en la distribución interna del presupuesto, aumentando a lo largo del periodo; 
puede observarse que alcanzó su punto máximo en el quinquenio, durante 1991.

Este subprograma recibe más de la mitad del presupuesto total de Investigación, porcentaje que es superior a la suma de las cantidades relativas recibidas por los Centros e Institutos de Investigación y el Sistema de Estudios de Posgrado. Le sucede en importancia presupuestaria el subprograma Centros e Institutos de Investigación (Cen. e Inst. Invest.), que no equivale a las dos terceras partes del anterior. Dentro de ese subprograma existen un total de 20 Centros e Institutos de Investigación (OPLAU, 1993), por lo cual resulta muy baja la proporción presupuestaria que se le destinó.

Los subprogramas Sistema de Estudios de Posgrado (SEP) y Empresas Auxiliares de Investigación (Emp. Aux. Invest.) tienen asignadas porciones muy reducidas, en comparación con los ya mencionados. El primero de estos muestra una tímida alza, mientras el segundo más bien disminuye a lo largo del quinquenio, especialmente en 1990. El subprograma Sedes Regionales solo aparece in- cluido en el presupuesto de Investigación como tal, en 1989.

El subprograma Apoyo a la Investigación comprende actividades relacionadas con: Evaluación a la Investigación (Eval. a Invest.), Servicios de Apoyo de Investigación (Serv.de Apoyo), Dirección Editorial y Difusión de la Investigación (Dir. Editorial), Oficina de Bibliotecas, Documentación e Información (Bibliotecas), Proyectos de Investigación (Proy. Invest.), Unidad de Microscopía Electrónica (Microsc. Electr.), Bioterio (Bioterio), Jardín Lankester (Jard. Lankester), Estaciones Experimentales Fabio Baudrit (E. F. Baudrit), Alfredo Volio (E. A. Volio) y Río Frío (E. Río Frio) y Laboratorio de Ensayos Biológicos (LEBI) (OPLAU, 1992). Posteriormente se incorporó además la Unidad de Desarrollo Tecnológico de la Información y Redes, que no aparece en el cuadro № 11 (UCR, 1993).

En ese cuadro № 11 se incluye la distribución porcentual del presupuesto asignado al subprograma Apoyo a la Investigación, en sus diferentes actividades.

\section{Cuadro 11}

Distribución porcentual anual del presupuesto del Subprograma Apoyo a la Investigación, en sus actividades, de 1988 a 1992

\begin{tabular}{|c|c|c|c|c|c|}
\hline Actividad & 1988 & 1989 & 1990 & 1991 & 1992 \\
\hline Eval. a Invest. & 0,86 & 0,53 & 0,17 & 0,16 & - \\
\hline Serv.de Apoyo & 30,83 & 29,88 & 29,35 & 37,45 & 32,12 \\
\hline Dir. Editorial & 5,18 & 5,32 & 5,96 & 4,99 & 4,98 \\
\hline Bibliotecas & 30,57 & 31,91 & 31,45 & 29,45 & 32,43 \\
\hline Proy. Invest. & 9,63 & 8,66 & 8,78 & 7,11 & 7,04 \\
\hline Microsc. Electr. & 2,16 & 1,91 & 1,89 & 1,67 & 2,00 \\
\hline Bioterio & 1,42 & 1,51 & 1,40 & 1,21 & 1,36 \\
\hline Jard. Lankester & 1,54 & 1,88 & 2,23 & 1,56 & 1,72 \\
\hline E. F. Baudrit & 13,16 & 13,60 & 13,81 & 12,00 & 13,55 \\
\hline E. A: Volio & 4,36 & 4,47 & 4,70 & 4,09 & 4,57 \\
\hline E. Río Frío & 0,16 & 0,16 & 0,14 & 0,12 & 0,10 \\
\hline LEBI & 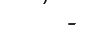 & 0,10 & 0,08 & 0,14 & 0,13 \\
\hline Nutr. Animal & 0,09 & 0,06 & - & - & - \\
\hline
\end{tabular}

Fuentes: OPLAU, 1988 b; 1989 b; 1990; $1991 ; 1992$. Elaboración propia. 
Cuadro 12

Proyectos inscritos por las unidades académicas en la Vicerrectoría de Investigación, en datos porcentuales, según su condición, de 1985 a junio de 1993

\begin{tabular}{lccrc}
\hline $\begin{array}{l}\text { Condición } \\
\text { del } \\
\text { proyecto }\end{array}$ & Fac. y Esc. & C. e Inst. Invest. & Sedes Reg. & Total \\
\hline & & & & \\
En desarrollo & 14,69 & 20,73 & 1,88 & 37,31 \\
Suspendidos & 1,61 & 1,67 & 0,06 & 3,35 \\
Cerrados & 2,48 & 3,08 & 0,20 & 5,77 \\
Terminados & 30,80 & 20,73 & 2,01 & 53.55 \\
\hline
\end{tabular}

Fuentes: Centro de Informática, 1993.

Elaboración propia.

En el subprograma Apoyo a la Investigación cobran mayor importancia relativa las actividades Servicios de Apoyo y la Oficina de Bibliotecas, Información y Documentación. En ambos casos, revisando los presupuestos respectivos (OPLAU, 1988 b; 1989 b; 1990; 1991; 1992), se observa que el pago de salarios al personal tuvo una cuota muy alta de participación. La primera de estas actividades también incluye servicios no personales como mantenimiento y reparación de máquinas, materiales y equipo, compra de libros, entre otras. En la segunda no se incluye la adquisición de libros $\mathrm{u}$ otros materiales didácticos, porque eso se contempla en la actividad Servicios de Apoyo.

Actividades tan importantes como Direc:ión Editorial y Proyectos de Investigación, İemás de tener poca importancia presupues--a. manifestaron un descenso, situación que Ea directamente a la labor académica in$\because \leq i z=$ iva.

- a idea de cómo los académicos se in$\therefore \ldots a$ en investigación, la da el análisis Ii: :-r vectos inscritos en la Vicerrectoría - oue aparecen agrupados según la ane se encontraban, desde 1985

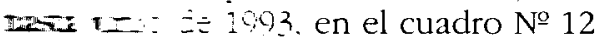

it $2=20$ mes ligados a Centros e Insti-

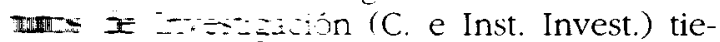
men a zación en proyectos en atr

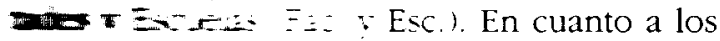
Pu $=2$ mavor porcentaje co-

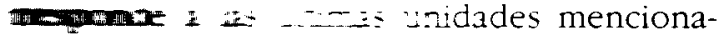

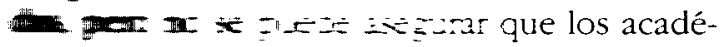

micos pertenecientes a ellas, hayan llevado a cabo, proporcionalmente, más proyectos puesto que cuentan con un número de miembros bastante mayor que los Centros e Institutos de Investigación.

Ya que la mayoría de los académicos está inscrita en la Vicerrectoría de Docencia, encontrándose ligados a una Escuela o Facultad y varios de ellos tal vez colaborando con algún Centro o Instituto de Investigación o presentan un proyecto como emanado de su unidad académica, no es posible establecer una separación tajante entre el personal académico de las Escuelas y Facultades y el de los Centros e Institutos de Investigación. Tampoco es posible determinar en cuáles de esas condiciones mencionadas anteriormente, se encuentran los investigadores participantes en los proyectos tabulados en el cuadro № 12.

Lo que si es claro es que la mayor parte de los proyectos en desarrollo provienen de los Centros e Institutos de Investigación, pudiendo establecerse entonces que para un académico fue más factible participar en algún proyecto de investigación cuando se encontraba ligado a un Centro o Instituto, que si no lo estuvo.

Para determinar cuál es la distribución de proyectos inscritos según el área académica de procedencia, se presenta el cuadro № 13

El mayor porcentaje de investigaciones provino del área de Ciencias Exactas y Naturales, siguiendo a una distancia considerable, aquellas emanadas de Ciencias Sociales y de Salud. 
Cuadro 13

Proyectos inscritos en la Vicerrectoría de Investigación, según el área de procedencia, en porcentajes, de 1985 a junio de 1993

\begin{tabular}{lr}
\hline \multicolumn{1}{c}{ Área } & $\%$ \\
\hline Ciencias Exactas y Naturales & 40,80 \\
Salud & 16,51 \\
Tecnológicas & 0,27 \\
Ingenierías y Arquitectura & 4,70 \\
Estaciones Experimentales & 1,48 \\
Humanística & 5,50 \\
Ciencias Sociales & 21,27 \\
Educación & 4,43 \\
Administración & 0,94 \\
Sedes Regionales & 4,10 \\
\hline
\end{tabular}

Fuentes: Centro de Informática, 1993.

Elaboración propia.

La mayor parte de la investigación que se realizó en el período estudiado en la UCR, fue en los campos de las Ciencias Exactas y Naturales, situación que pudo estar determinada por el procedimiento seguido en la Vicerrectoría de Investigación para aprobar un proyecto. (Veáse el "Formulario para la valoración de propuestas de investigación sobre criterios de prioridad", elaborado por la Vicerrectoría de Investigación; en él se nota un sesgo a favor de las investigaciones en el área de las ciencias exactas y naturales) (UCR, 1992).

Tal situación revela intereses institucionales de favorecer la producción de conocimientos en ciertos campos del saber, a costa de otros. Ello se debe principalmente a la limitación de recursos destinados a la investiga- ción, lo cual ha obligado al Alma Mater a priorizar áreas de investigación de acuerdo a su productividad potencial.

Desde la perspectiva del académico entonces, hubo mayores posibilidades de inscribir un proyecto en la Vicerrectoría de Investigación, y por lo tanto participar en esa actividad, si estaba ligado a un Centro o Instituto de Investigaciốn y si además era del área de Ciencias Exactas y Naturales.

A partir del análisis del cuadro $\mathrm{N}^{\mathrm{Q}} 14$ se trata de determinar, al igual que como se hizo con el programa de Docencia, la importancia relativa que se concedió a los diferentes tipos de empleados universitarios pertenecientes al programa de Investigación.

En este programa es notable el descenso experimentado por la participación del grupo docente (D) de 1988 a 1989 , sin que se retorne a la situación inicial, a lo largo del período. Simultáneamente se produce un aumento a más del doble por parte de los administrativos, que aunque desciende en años sucesivos, siempre se mantiene en cifras superiores al $45 \%$. El personal de apoyo académico también bajó notablemente en esa época su participación porcentual, siguiendo una ruta muy parecida a la de los docentes, aunque de principio a fin, contó con cuotas más elevadas que ellos.

Ya que la mayoria del personal docente está inscrito en el Programa de Docencia, no es sorprendente que sus porcentajes sean tan bajos en investigación. Lo que si llama la atención es que aumentara tanto el porcentaje del presupuesto destinado al pago de salarios administrativos, a costa del descenso en

Cuadro 14

Distribución porcentual anual del presupuesto de Investigación correspondiente al pago de salarios, según los tipos de empleados

\begin{tabular}{cccccc}
\hline Personal & 1988 & 1989 & 1990 & 1991 & 1992 \\
\hline D & 31,22 & 20,47 & 22,80 & 21,74 & 21,90 \\
A & 21,83 & 49,89 & 46,95 & 46,33 & 35,73 \\
AA & 46,95 & 29,62 & 30,24 & 31,91 & 32,35 \\
A + AA & 68,78 & 79,51 & 77,19 & 78,24 & 78,08 \\
\hline
\end{tabular}

Fuentes: OPLAU, 1988 b; 1989 b; 1990; 1991; 1992.

Elaboración propia. 
Cuadro 15

Distribución anual del presupuesto de Acción Social por subprogramas, en datos porcentuales, de 1988 a 1992

\begin{tabular}{lrrrrr}
\hline Subprograma & 1988 & 1989 & 1990 & 1991 & 1992 \\
\hline & & & & 43,98 & 41,40 \\
Divulg. y Difus. & 47,13 & 45,26 & 49,75 & 50,28 & 5,73 \\
Apoyo a Ac.Soc. & 45,69 & 49,99 & 45,00 & 5,24 & 5,69 \\
Empr. Aux. Ac. Soc. & 7,17 & 4,75 & & \\
\hline
\end{tabular}

Fuentes: OPLAU, 1988 b; 1989 b; 1990; 1991; 1992.

Elaboración propia.

los correspondientes a docentes y apoyo académico.

Como puede verse en el último renglón del cuadro en análisis, en el primer año del período estudiado, 1988, la participación del personal docente era mayor, pero luego decayó. Se observa que en los cuatro años siguientes la relación $\mathrm{D} / \mathrm{A}+\mathrm{AA}$ está invertida con respecto a la del Programa de Docencia (comparar los cuadros №14 y № 9).

Se repite en Investigación lo señalado anteriormente: que se da un incremento en el presupuesto destinado al aparato administrativo a costas del académico, sobre todo en un programa donde la participación del cuerpo docente debió ser más vasta para que la Universidad cumpliera a cabalidad con las funciones que le encomienda el Estatuto Orgánico.

No es posible determinar a partir de los datos anteriores, cuál es la participación real de los académicos en el presupuesto de Investigación, porque la mayoría se incluyen en el presupuesto de Docencia.

A partir del análisis realizado, se puede asegurar que la Vicerrectoría de Investigación no contó con los recursos financieros apropiados para poder impulsar y apoyar en forma debida, programas y proyectos inter y multidisciplinarios, no solo en el área de las Ciencias Exactas y Naturales sino en todas las áreas del conocimiento.

Se fue mermando el apoyo a la publicación y difusión de los conocimientos adquiridos por medio de investigaciones. El crecimiento del aparato administrativo en el Programa de Investigación revela, al igual que en el de Docencia, que los fondos no se destina- ron con prioridad a los sectores directamente involucrados con la labor investigativa, puesto que el aumento en el presupuesto dedicado a administración de la investigación se dió a costas de la parte académica, situación poco estimulante para los docentes.

\section{II. c. Vicerrectoria de Accion Social}

La Vicerrectoría de Acción Social es un organismo de colaboración inmediata con la Rectoría. Constituye el vínculo primordial entre la UCR y el país, ya que es la instancia encargada de organizar, coordinar, dirigir, promover y evaluar todas las actividades de extensión, divulgación y trabajo comunal (CEA, 1981).

Para cumplir sus objetivos la Vicerrectoría organiza su quehacer en dos subsistemas a saber: Comunicación-Información e Interacción Socioeducativa (Vicerrectoría de Acción Social, 1993).

Siguiendo el mismo procedimiento que se aplicó a los dos Programas anteriores, se analiza a continuación cómo se distribuye el presupuesto de Acción Social por subprogramas, para lo cual se elabora el cuadro № 15 .

El programa de Acción Social repartió la mayor parte de su presupuesto entre los subprogramas de Divulgación y Difusión (Divulg. y Difus.) y Apoyo a la Acción Social (Apoyo a Ac. Soc.), concediéndole un pequeño porcentaje a Empresas Auxiliares de Acción Social (Empr. Aux. Ac. Soc.).

Analizando detalladamente la subdivisión que se hizo, por subprogramas, se observa que 
en 1988 Divulgación y Difusión tenía mayor importancia relativa que Apoyo a la Acción Social, pero conforme avanza el quinquenio la situación cambia, excepto en 1990 .

Pareciera que se presenta en este programa el mismo proceso que en los dos previamente estudiados. Con la intención de corroborar esa idea, se analiza el presupuesto de Acción Social destinado al pago de salarios, según el personal empleado, con ayuda del cuadro № 16 .

\section{Cuadro 16}

Distribución porcentual anual del presupuesto de Acción Social correspondiente al pago de salarios, según los tipos de empleados

\begin{tabular}{crrrrr}
\hline Personal & 1988 & 1989 & 1990 & 1991 & 1992 \\
\hline & & & & & \\
D & 6,45 & 7,32 & 1,39 & - & 5,04 \\
A & 87,46 & 24,04 & 24,84 & 23,61 & 22,82 \\
A A & 6,07 & 68,63 & 73,75 & 76,39 & 72,13 \\
A + AA & 95,53 & 92,67 & 98,59 & 100 & 94,95 \\
\hline
\end{tabular}

Fuentes: OPLAU, 1988 b; 1989 b; 1990; 1991; 1992. Elaboración propia

Como ya se había establecido, este es el programa que agrupa al menor porcentaje de docentes (D) de la Universidad, de ahí que también la proporción del presupuesto destinado a ellos sea muy baja, llegando a ser nula en 1991. Entre el personal administrativo (A) y el de apoyo académico (AA) llama la atención que de 1989 en adelante las proporciones se modifican con respecto a 1988 .

No fue posible conseguir explicación a ese fenómeno, en la Vicerrectoría respectiva. La situación hace pensar una vez más, que se recurrió al nombre de apoyo académico para justificar el crecimiento del personal encargado de las labores administrativas o que hubo recalificación y cambio de funciones dentro del personal administrativo a cargo del Programa de Acción Social. Se observa en el último renglón del cuadro $\mathrm{N}^{\circ} 16$, que casi la totalidad del presupuesto para el pago de salarios en este Programa, y en un 100\% durante 1991, fue destinado a empleados que no realizan labores académicas.
Según se había indicado al inicio (ver cuadro № 2), la proporción del presupuesto universitario correspondiente a Acción Social fue casi la más baja de todas, lo cual indica la poca importancia que la Universidad concedió a este Programa, a pesar de cumplir ella una de las tres funciones básicas del Alma Mater según el Estatuto Orgánico.

Varios funcionarios de esa Vicerrectoría (Ålvarez, 1993; Chen, 1993; Hernández, 1993) concordaron en que el presupuesto recibido ha sido insuficiente para desarrollar los programas propuestos, de ahí que dicha instancia explore nuevas vías para captar financiamiento externo. Además indicaron que gran parte de sus recursos financieros se han empleado en solucionar problemas inesperados, como la reparación de daños causados por desatres naturales, que merman los recursos ya asignados a los diferentes proyectos. Por otra parte, también esa Vicerrectoría se ha abocado a la búsqueda de mecanismos de estímulo para que los docentes se interesen más en la Acción Social (Vicerrectoría de Acción Social, 1993).

Tal situación deja entrever, por una parte, que existe gran flexibilidad en el manejo del presupuesto, que afecta lo programado. Por otra parte, que este es el programa al que se recurre cuando surgen gastos inesperados en la Institución.

Todo ello refuerza la creencia de que a la Acción Social no se le ha concedido la importancia, que de acuerdo con lo establecido, debería darle la UCR. Además, dentro de ese papel insignificante que se le ha asignado a la Vicerrectoria, el personal docente tiene una importancia relativa también mínima.

\section{Conclusiones}

Un análisis somero de los presupuestos universitarios y su distribución, a lo largo del quinquenio en estudio, revela que sus montos se mantuvieron siempre en avance continuo, superior a la tasa de inflación.

No puede asegurarse que la UCR haya sido presupuestariamente afectada por el Ajuste Estructural costarricense, de 1988 a 1992. Por el contrario, más bien parece haberse incrementado la burocracia. Los datos revelan que disminuyó la importancia relativa 
del personal docente, mientras aumentó progresivamente la del personal dedicado a labores administrativas. Para sostener ese crecimiento se realizaron modificaciones en el presupuesto, favorables a la parte administrativa y desfavorables a la docente.

Sólo una porción que representa poco más de la mitad del presupuesto universitario, se destinó a los programas académicos -docencia, investigación y acción social-. De esa parte, sólo una fracción muy reducida fue aplicada a la labor estrictamente académica, pues el sector admnistrativo consumió el mayor porcentaje.

Puede concluirse que en la UCR, a lo largo del lustro analizado, se favoreció el sector burocrático-administrativo, en detrimento de lo académico propiamente dicho.

El estudio de los tres programas dedicados a satisfacer las funciones básicas de la Universidad -docencia, investigación y acción social- no evidenció cambios en el orden de importancia concedido a las diferentes áreas académicas. La Vicerrectoría de Docencia continuó dando la misma importancia relativa a los subprogramas académicos, durante el quinquenio. Ello sugiere que hubo muy pocas modificaciones en ese sentido, para adaptar la Universidad a las nuevas corrientes ideológizas neoliberales.

La situación creada por el crecimiento continuo, tanto del presupuesto como del apa:ato burocrático-administrativo, junto a un zrograma de docencia que no cambió sus zondades, indican que la Universidad, a pe¿- Le las modificaciones introducidas en dife$\because=-$ s niveles de las instituciones públicas ancenses por los Programas de Ajuste Es- - - enio estudiado. Esa falta de adaptación

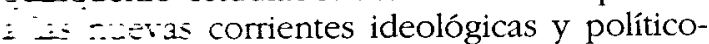
- a s ha venido relegando a un segunI: $=:-1$ cuerpo docente y con él su misión a destirtuándose la misión primor$=-1.5$ Estatuto Orgánico le confiere a la $\because=12 \pm \vdots$ de Costa Rica.

\section{Diografia}

15. Teresa: Vicerrectoría de Acción - : = ersidad de Costa Rica. Inforrazoral 1993.
BANCO Central de Costa Rica. Departamento Indice de Precios, mimeografiado, 1994.

CAMACHO, Daniel: "La inacabada lucha pro académica del III Congreso Universitario". Revista de Ciencias Sociales, UCR № 49-50, 1990: 63-76.

CEA (Centro de Evaluación Académica de la UCR): Distribución de la carga académica de los docentes, mimeografiado, 1993.

Fascículo de Información General Universidad de Costa Rica. UCR, 1981.

Centro de Informática de la UCR: Proyectos de investigación inscritos en la Vicerrectoria de Investigación, mimeografiado, 1993.

COMISION de Enlace (Actas): Fondo Especial de la Educación Superior y Asignaciones Especiales otorgadas a las instituciones de educación superior universitaria estatal 1981-1983. San José, División de Sistemas, mimeografiado, s.f.

CONARE (Consejo Nacional de Rectores): Aspectos financieros relacionados con las instituciones de educación superior universitaria estatal. San José, Oficina de Planificación de la Educación Superior, 1990

Estadistica de la educación superior 1991. San José, Oficina de Planificación de la Educación Superior, 1992.

: "Fórmula de cálculo del FEES". Acta de la reunión del 1 de noviembre de 1991, mimeografiado, 1991.

: Leyes, decretos y convenios relacionados con el financiamiento de la educación superior universitaria estatal de Costa Rica. San José, Oficina de Planificación de la Educación Superior, 1989.

CHEN, Gustavo: Vicerrectoría de Acción Social Universidad de Costa Rica. Información personal, 1993.

HERNANDEZ, Manuel: Vicerrectoria de Acción social, UCR. Información personal, 1993. 
La Gaceta № 126 Imprenta Nacional, lunes 28 de noviembre de 1988.

OCU - UCR (Oficina de Contraloría Universitaria): Relación bistórica en cuadros y grâficos. UCR, mimeografiado, 1990.

: Relación bistórica en cuadros $y$ gráficos y comentarios. UCR, mimeografiado, 1992.

OPLAU - UCR (Oficina de Planificación Universitaria): "Cuadro № 7: presupuesto de egresos por programas y subprogramas 1989-1993". Presupuesto 1993. UCR, mimeografiado, 1993.

: Panorama Cuantitativo Universitario 1985-1986. UCR, 1989 a.

: Presupuesto por programas y actividades 1988. UCR, $1988 \mathrm{~b}$.

Presupuesto por programas y actividades 1989. UCR, 1989 b.

: Presupuesto por programas y actividades 1990. UCR, 1990.

: Presupuesto por programas y actividades 1991. UCR, 1991.

: Presupuesto por programas y actividades 1992. UCR, 1992.

REIMERS, Fernando: "El costo educativo de la deuda externa. Implicaciones para la planificación educativa en América Latina". La Educación: Revista Interamericana de Desarrollo Educativo. Dpto. Asuntos Educativos de la OEA, Washington, Año XXXIII, No 105, 1989: 1-37.
RODRIGUEZ, Roberto: La universidad latinoamericana contemporánea. Los desafíos de la post-crisis. Universidad Nacional Autónoma de México. Centro de Estudios sobre la Universidad, mimeografiado, 1992.

ROJAS, Yolanda: “Transformaciones recientes en la educación costarricense". J. M. Villasuso (editor): El nuevo rostro de Costa Rica. Centro de Estudios Democráticos de América Latina, CEDAL. Heredia, Costa Rica 1992: 97-122.

SANCHEZ, Oscar: Oficina de Planificación Universitaria UCR. Información personal, 1993.

UNIVERSIDAD de Costa Rica, Asamblea Universitaria: Acuerdos Definitivos originados en el III Congreso Universitario 19711972 UCR, 1979.

UNIVERSIDAD de Costa Rica: Estatuto Orgänico. Oficina de Publicaciones de la UCR, Ediciones de 1984 y 1990.

: Formulario para la valoración de propuestas de investigación sobre criterios de prioridad. Vicerrectoria de Investigación, mimeografiado, 1992.

: "Reglamento de Régimen Académico y Servicio Docente "Reglamentos de la Universidad de Costa Rica. Edición Actualizada al 11 de Agosto de 1993, mimeografiado, 1993.

VICERRECTORIA de Acción Social, UCR: La Acción Social: conceptualización y organización, UCR, 1993. 